\title{
Article
}

\section{The effect of cut-off frequency when high-pass filtering equine sEMG signals during locomotion}

St George, Lindsay Blair, Hobbs, Sarah Jane, Richards, James, Sinclair, Jonathan Kenneth, Holt, D. and Roy, S.H.

Available at http://clok.uclan.ac.uk/24170/

St George, Lindsay Blair ORCID: 0000-0002-5531-1207, Hobbs, Sarah Jane ORCID: 0000-0002-1552-8647, Richards, James ORCID: 0000-0002-40043115, Sinclair, Jonathan Kenneth ORCID: 0000-0002-2231-3732, Holt, D. and Roy, S.H. (2018) The effect of cut-off frequency when high-pass filtering equine SEMG signals during locomotion. Journal of Electromyography and Kinesiology, 43 . pp. 28-40. ISSN 1050-6411

It is advisable to refer to the publisher's version if you intend to cite from the work. http://dx.doi.org/10.1016/j.jelekin.2018.09.001

For more information about UCLan's research in this area go to

http://www.uclan.ac.uk/researchgroups/ and search for <name of research Group>.

For information about Research generally at UCLan please go to http://www.uclan.ac.uk/research/

All outputs in CLoK are protected by Intellectual Property Rights law, including Copyright law. Copyright, IPR and Moral Rights for the works on this site are retained by the individual authors and/or other copyright owners. Terms and conditions for use of this material are defined in the policies page.

\section{CLoK}

Central Lancashire online Knowledge www.clok.uclan.ac.uk

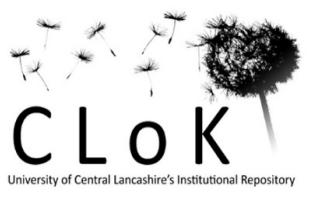


The effect of cut-off frequency when high-pass filtering equine sEMG signals during locomotion

St. George, L. ${ }^{1}$, Hobbs, S. J. ${ }^{1}$, Richards, J. ${ }^{2}$, Sinclair, J. ${ }^{1}$, Holt, D. ${ }^{3}$, Roy, S.H. ${ }^{4}$

${ }^{1}$ University of Central Lancashire, Centre for Applied Sport and Exercise Sciences, Preston, PR1 2HE, 5 United Kingdom;

${ }^{2}$ University of Central Lancashire, Allied Health Research Unit, Preston, PR1 2HE, United Kingdom;

${ }^{3}$ Department of Research, Myerscough College, Preston, UK

${ }^{4}$ Delsys Inc., Natick, MA

Corresponding author:

Lindsay St. George, Centre for Applied Sport and Exercise Sciences, University of Central Lancashire.

Preston, PR1 2HE, United Kingdom.

Tel: 01772893775

Email: lbstgeorge@uclan.ac.uk

15

Keywords: equine, surface electromyography, high-pass filter, movement artefact, gait.

\section{Conflict of Interest:}

None

\section{Sources of Funding:}

25 None 


\section{Introduction}

The use of surface electromyographic (sEMG) signals in equine biomechanics research represents a growing area of interest. sEMG has primarily been used to understand muscle function during normal equine locomotion, but has also been used to investigate the effect of aging (Zsoldos et al., 2014), lameness (Zaneb et al., 2009), dental treatment (Williams et al., 2014) and back stiffness (Peham and Schobesberger, 2006) on equine muscle function. Unfortuantely, variation in the methods used for signal detection and processing in equine sEMG literature is problematic, making comparisons between studies difficult (Valentin and Zsoldos, 2016). As such, equine researchers are advised to follow the path of human sEMG, where standardised guidelines have been developed for sensor characteristics, muscle location, as well as signal detection, conditioning, and analysis (Winter et al., 1980; De Luca, 1997; Stegeman and Hermens, 1998; Merletti, 1999; De Luca et al., 2010). A similar framework is needed for best practices in equine sEMG, where the unique challenges associated with detecting and processing sEMG data from equine subjects are considered. Valentin and Zsoldos (2016) outline these challenges, which include, but are not limited to: skin preparation for coats with excessive hair, dirt and oil, behavioural constraits and the location of sensors on larger muscles. These challenges restrict the use of human sEMG techniques in horses and establishing best practice guidelines for equine sEMG studies is therefore required. In recognition of this, we have initiated this development with a study that focuses solely on signal processing methods.

Standards for human sEMG signal processing require the removal of low-frequency noise sources, generally accomplished using high-pass filtering (HPF) (Merletti, 1999; De Luca et al., 2010). The low-frequency component of the sEMG frequency spectrum is highly susceptible to contamination from baseline and movement artefact noise sources, particularly when the signal is obtained during dynamic movements where electromechanical disturbances to the electrode-skin interface are expected (De Luca et al., 2010). These noise sources introduce modifications to the raw signal that can alter one's ability to interpret the data accurately. This is especially problematic for equine research, as the weight, speed and gait characteristics of horses will result in greater impact forces and resultant pertubations to the electrode-skin interface. Thus, a greater dependency on optimal filtering is likely for equine sEMG 
signals. However, a recent systematic review revealed that most animal sEMG studies fail to report, or do not describe, the use of HPF to remove low-frequency noise (Valentin and Zsoldos, 2016). In an effort to develop standardised guidelines for equine sEMG signal processing, research should develop best practice in a systematic manner that follows the incremental signal processing steps outlined in human guidelines. Thus, the determination of optimal HPF techniques, offers a systematic starting point for informing equine sEMG signal processing guidelines and will form the focus of this study.

The development of standardised HPF guidelines requires consideration of multiple factors, including filter type, filter order, types of low-frequency noise sources and the cut-off frequency required to attenuate these sources. In human literature, the determination of optimal HPF cut-off frequency has received considerable attention (Van Boxtel et al., 1998; Van Boxtel, 2001; Brown et al., 2009; Potvin and Brown, 2004; De Luca et al., 2010) and has been described as a compromise between reducing noise and artefact contamination, while preserving the desired muscle activation information from the signal (Winter, 2009; De Luca et al., 2010). In human research, qualitative comparisons of the power spectra of HPF signals (Van Boxtel et al., 1998; Van Boxtel, 2001; De Luca et al., 2010) and quantitative comparison of signal loss values, calculated using the RMS envelope of HPF signals (De Luca et al., 2010), are generally used to determine the attenuation rates of movement artefact and sEMG signal components, as a function of cut-off frequency. These studies recommend the use of a HPF cut-off frequency $\geq 20 \mathrm{~Hz}$ for most applications (Van Boxtel et al., 1998; Van Boxtel, 2001; De Luca et al., 2010). In equine sEMG research, HPF cut-off frequencies of $10 \mathrm{~Hz}$ (Harrison et al., 2012), $20 \mathrm{~Hz}$ (Hodson-Tole., 2006; Crook et al., 2010; Harrison et al., 2012) and 40 Hz (Kienapfel, 2015; Kienapfel et al., 2018) have been reported. No known studies have employed the methods described in human literature to determine the optimal HPF cut-off frequency for equine sEMG signals. Furthermore, while the energetic component of the sEMG signal containing low-frequency noise sources has been wellstudied in human recordings, the same cannot be said for equine data. Therefore, an investigation of HPF cut-off frequency may serve to provide an initial understanding of low-frequency noise contamination in equine sEMG signals. From here, future research could examine other factors, such as optimal filter type and order, which are required for the development of standardised guidelines. 
This study aims to determine the optimal HPF cut-off frequency for attenuating movement artefact noise from equine sEMG signals. We adopted an approach similar to that described in human studies (Van Boxtel et al., 1998; Van Boxtel, 2001; De Luca et al., 2010) where sEMG signals are HPF with various cut-off frequencies, subjected to power spectral analysis and enveloped using RMS to calculate spectral peaks, indicative of motion artefact, and signal loss, respectively. Optimal cut-off frequency is then determined by comparing the attenuation rates of sEMG signals, based on visual examination of power spectra and statistical analysis of RMS signal loss data, as a function of cut-off frequency. In an effort to understand the effect of equine gait on the generation of movement artefact noise, sEMG signals are obtained from the Triceps Brachii (long head) (TB) and Biceps Femoris (vertebral head) (BF) during trot and canter. BF and TB represent large muscles from the equine forelimb (FL) and hindlimb (HL), respectively, and were chosen due to their differing size, thickness and function (Payne et al., 2005a,b). sEMG signals are further segmented into kinematic stance and swing phases because of reported differences in skin displacement noise (van Weeren et al., 1990a, b; Hjerten et al., 1994). It is hypothesised that equine sEMG signals will exhibit low-frequency noise contamination that will vary depending on muscle and gait. Due to the highly dynamic nature and high vertical forces reported during the equine gaits studied (Merkens et al., 1993a,b), it is also hypothesised that the sEMG signals studied will require a higher cut-off frequency than those reported in human literature. As the first study of its kind, its intent is to quantify noise contamination of the low-frequency component of the equine sEMG spectrum and demonstrate the importance of HPF in equine sEMG signal processing. Thus, it is envisaged that findings from this study will form a preliminary framework and should be treated as a starting point for future research to develop comprehensive guidelines for equine sEMG signal processing.

\section{Methods}

Ethical approval was obtained from the University of Central Lancashire’s Animal Projects Committee (RE/13/04/SH, RE/13/01/SH). Written informed consent was obtained from all 
horse owners, riders and handlers. Due to the preliminary nature of this work, data from existing data sets were employed, where data were collected for three separate studies exploring the kinematics and muscle activity patterns of trot and canter. The same data collection techniques were employed by the same researchers across all studies, but different equine subjects were used for each study.

\section{Horses}

Data were collected from 20 horses (age: $11.2 \pm 3.7$ years, height: $153.9 \pm 8.9 \mathrm{~cm}$, sex: 12 geldings, 8 mares, breed: various). All horses were in training and free from lameness, as defined by their owner. Data were collected from 10 horses during in-hand trot, and from 10 horses during ridden canter. Horses were ridden by riders with similar experience and ability. Unridden horses were trotted in-hand by one experienced handler.

\section{Equipment set-up, skin preparation and sensor fixation}

sEMG data were collected from BF and TB using wireless sEMG sensors (Trigno ${ }^{\mathrm{TM}}$, Delsys Inc., USA), with a bi-polar parallel bar electrode configuration and an inter-electrode distance of $10 \mathrm{~mm}$. Prior to data collection, all hair was removed from electrode sites. Sensor sites for BF were approximately halfway between the third trochanter and patella, and approximately $9 \mathrm{~cm}$ cephalad to the cranial margin of Semitendinosus (Schuurman et al., 2003) (Figure 1a). Sensor sites for TB were midway between the olecranon and proximal point of scapular spine, measured at an angle of 50 degrees from a line drawn between the olecranon and intermediate tubercle of humerus (Hodson-Tole, 2006) (Figure 1b). Following warm-up, sensor sites were thoroughly cleaned using isopropyl alcohol wipes. A small amount of saline solution was applied to the electrode bars to act as an electrolytic solution (Cram and Rommen, 1989; Clancy et al., 2002). Sensor adhesion was achieved using a combination of Delsys Adhesive Surface Interface strips (Delsys Inc., USA) and strips of double-sided tape, which were applied to the top and bottom of the sensor next to each electrode pair. The sensor was positioned on 
the muscle belly so that the electrode bars were oriented perpendicular to the underlying muscle fibre direction (De Luca, 1997; Hermens et al., 2000).

Three-dimensional kinematic data were collected using eight Qualisys Oqus cameras (Qualisys AB, Sweden), positioned side-by-side in a linear configuration. An extended calibration was conducted to collect data from multiple strides and produced a calibration volume approximately $8 \mathrm{~m}$ in length.. Spherical retro reflective markers, (25 mm diameter) were positioned over anatomical landmarks as described in Holt et al., 2017 and Figure 1 using double-sided tape.

Insert Figure 1

\section{Data Collection}

Data were collected using Qualisys Track Manager (QTM) software (Qualisys AB, Sweden). Kinematic (232 Hz) and sEMG (2088 Hz) data were synchronously acquired using an external trigger system (Delsys Trigger Module, Delsys Inc., USA). A static trial was first recorded from each horse, which then progressed through the capture volume during in-hand trot or ridden canter. Riders and handlers were instructed to position horses adjacent to placing poles, positioned approximately $4.5 \mathrm{~m}$ from the cameras to demarcate the optimal capture volume. A minimum of six trials were collected from each horse. Three canter trials were collected from the left and right lead. Horses were permitted to travel at their preferred velocity.

\section{Data processing and analysis}

Raw sEMG signals were differentially amplified by a factor gain of 909, a CMRR of $>80 \mathrm{~dB}$ and an internal Butterworth high-pass (20 $\pm 5 \mathrm{~Hz}$ cut-off, $>40 \mathrm{~dB} / \mathrm{dec}$ ) and low-pass filter (450 $\pm 50 \mathrm{~Hz}$ cutoff, >80 dB/dec). Kinematic data were tracked in QTM and imported into Visual3D (C-Motion Inc., USA) for further analysis. Kinematic data were interpolated and low-pass filtered (Butterworth $4^{\text {th }}$ order), with a cut-off frequency of $12 \mathrm{~Hz}$, as determined using residual analysis. FL and HL hoof impact and lift-off events were calculated from kinematic data in accordance with Holt et al. (2017), using the FL and HL sagittal plane angles illustrated in Figure 1. Angle-time curves were plotted for FL and HL 
sagittal plane angles. An angle of zero degress was observed when the two segments were aligned and was used as a threshold for gait event detection for the FL and HL, with hoof impact and lift-off coinciding with descent and ascent and through zero degrees, respecitively (Holt et al., 2017). Kinematic gait events were applied to sEMG signals to segment the signal into stance and swing phase (Figure 2). TB and BF were chosen to represent FL and HL muscles, respectively. Therefore, FL gait events were applied to TB signals and HL events applied to BF to ensure that muscle activity was directly related to the kinematics of the associated limb. A constant delay of $20 \mathrm{~ms}$ between kinematic and sEMG data was corrected for by shifting sEMG signals forward by 5 frames prior to applying kinematic gait events. To calculate stride velocity, the first derivative of the croup marker was calculated and the average velocity was calculated between consecutive HL impact events.

$$
\text { Insert Figure } 2 .
$$

\section{High Pass Filtering of Raw sEMG Signals}

sEMG data from individual stance and swing phases were exported from Visual3D into Delsys EMGworks (Delsys Inc., USA) for further analysis. Due to the pre-existing nature of the data set, data from each muscle and gait combination were not available across all horses. Therefore, from the group of 20 horses, data from five different horses were analysed for each of the four muscle and gait combinations investigated (TB trot $(n=5)$, TB canter $(n=5)$, BF trot $(n=5)$, BF canter $(n=5))$. No horse contributed to more than one group. sEMG signals from stance and swing phase were DC offset removed and, in accordance with (Kienapfel, 2015; Kienapfel et al., 2018), were HPF using a Butterworth $4^{\text {th }}$ order filter. HPF cut-off frequencies ( $n$ ranging between $10-80 \mathrm{~Hz}$ ), were applied to each signal in $10 \mathrm{~Hz}$ increments. An unfiltered signal was retained for each signal, resulting in nine representative sEMG signals for each stance and swing phase.

\section{Power Spectral Analysis and RMS Filtering of Signals}

The power spectral density of each HPF signal was calculated using a Hanning window (Fast Fourier Transformation (FFT) length: $0.25 \mathrm{~s}$, window length: $0.125 \mathrm{~s}$, window overlap: $0.0625 \mathrm{~s}$ ). HPF signals 
window overlap: $0.03 \mathrm{~s}$ ). FFT and RMS window length were based on average stance and swing duration of $0.32 \pm 0.05 \mathrm{~s}$ and $0.32 \pm 0.04 \mathrm{~s}$, respectively, across all horses and gaits.

\section{Calculation of Signal Loss (\%)}

In accordance with De Luca et al. (2010), mean ( \pm SD) signal loss (\%) was calculated using the mean RMS value from each signal. Signal loss (\%) was calculated in accordance with equation 1 . Residual signal (\%) was used as a method for examining attenuation rates and was calculated in accordance with equation 2. Figure 3 shows an example of signal loss and residual signal (\%) data from one stance and swing phase signal.

200

1. Signal Loss $(\%)=\frac{\left(R M S_{\text {full }}-R M S_{h p f}\right)}{R M S_{f u l l}}$

2. Residual Signal (\%) $=\frac{R M S_{h p f}}{R M S_{\text {full }}}$

Where $\mathrm{RMS}_{\text {full }}$ is the mean RMS value of the HPF unfiltered signal ( $3 \mathrm{~dB}$ filter bandwidth $=0 \mathrm{~Hz}$ to $400 \mathrm{~Hz}$ ) and $\mathrm{RMS}_{\mathrm{hpf}}$ is the mean RMS value of the HPF filtered signal (3 dB filter bandwidth $=\mathrm{n} \mathrm{Hz}$ to $400 \mathrm{~Hz}$, where $n=10,20,30,40,50,70,80$ )

Insert Figure 3.

\section{Analysis of Processed Signals}

Qualitative and quantitative analysis were performed to examine the effect of HPF cut-off frequency on sEMG signal quality, power spectra and RMS signal loss. Residual signal loss is inversely proportional to RMS signal loss values, thus statistical analyses were performed on signal loss data only, which were tested for normality using Shapiro-Wilks tests. Repeated measures one-way ANOVAs were employed to compare the effect of increasing cut-off frequency increments $(10 \mathrm{~Hz})$ on RMS signal loss data from each muscle and gait. Significance was identified at $P<0.05$ and post hoc pairwise comparisons were conducted using a Bonferroni correction where significant main effects were found. Qualitative and quantitative findings were used to provide empirical evidence for which filter setting 
elicited the highest signal quality through reduction of low-frequency noise sources, while maximally retaining the true sEMG signal (De Luca et al., 2010).

\section{Results}

A total of 236 stance and 184 swing phases were analysed across both gaits and muscles. Stride velocity for trot and canter were $3.4 \pm 0.5 \mathrm{~m} / \mathrm{s}$ and $4.7 \pm 0.5 \mathrm{~m} / \mathrm{s}$, respectively across all horses executing each gait. The effects of different HPF cut-off frequencies on representative sEMG signals, power spectra and RMS signals are presented in Figures $4-7$ and demonstrate that, as cut-off corner frequency increased, the amplitude of the raw sEMG and RMS signals decreased. Visual and quantitative inspection of RMS data revealed that signals began to conform when cut-off frequencies $\geq 30 \mathrm{~Hz}$ were applied, exhibiting corresponding activity patterns and incremental decreases in amplitude (Figures 4 7c, e). As cut-off frequency increased beyond the $40-50 \mathrm{~Hz}$ range, the amplitude of the signals continued to decrease, but to a noticeably lesser degree. Visual inspection of frequency spectra revealed a consistent low-frequency peak between $0-20 \mathrm{~Hz}$, which was fully attenuated when cut-off frequencies of $30-40 \mathrm{~Hz}$ were applied. This pattern was visually observed across all stance and swing phase data from both muscles and both gaits. (Figures $4-7 b, d$ ).

Mean \pm sd signal loss (\%) and residual signal loss (\%) values for each $10 \mathrm{~Hz}$ cut-off frequency increment across all muscles and gaits are illustrated in Figure 8. Pairwise comparisons are presented in Table 1 and show that increasing cut-off frequency intervals $(10 \mathrm{~Hz})$ resulted in significant increases in signal loss $(P<0.05)$ across the majority of muscles and gaits. The greatest rate of signal loss was generally exhibited between $10-30 \mathrm{~Hz}$ increments, with significant increases $(P<0.05)$ in signal loss observed between $20-30 \mathrm{~Hz}$ across all muscles and gaits. Non-significant changes in signal loss were mainly observed between $10-20 \mathrm{~Hz}$, apart from BF during canter and TB during trot swing phase. Increases in cut-off frequency $>30 \mathrm{~Hz}$ produced lower, but mainly significant, average signal losses of 2.0-9.5\% between each $10 \mathrm{~Hz}$ increment across all muscles and gaits, apart from $70-80 \mathrm{~Hz}$ cut-off frequency intervals, which were largely non-significant. For BF canter stance data (Figure 8b), a comparatively 
lower, but significant, average signal loss of $7.3 \pm 1.4 \%$ was found across all $10 \mathrm{~Hz}$ increments and resulted in a relatively linear increase in signal loss with increasing cut-off frequency increment. Expectedly, mean \pm sd residual signal loss (\%) data is a mirror image of the signal loss curve, which subsequently intersect at 50\% when residual and signal loss are equal (Figure 8). Ultimately, this intersection provides a quantitative representation of the point where the amount of attenuated signal is equal to the amount of remaining signal following HPF. When considered as a function of cut-off frequency, an intersection point at $30-40 \mathrm{~Hz}$ was observed for the majority of conditions, apart from TB swing phase and BF stance phase data during canter, which intersected between $20-30 \mathrm{~Hz}$ and 70 $-80 \mathrm{~Hz}$, respectively.

\section{Insert Figures $4-8$, Table 1}

\section{Discussion}

In this study, data from 20 horses of varying age, size, breed and training level were employed to produce a comprehensive dataset of over 400 sEMG signal recordings. Two muscles from the equine FL and HL, with differing size, thickness and function (Payne et al., 2005a,b) were investigated to further diversify the dataset. It was hypothesised that these variables, along with the known kinematic and kinetic differences between gaits, would elicit varying magnitudes of low-frequency noise contamination in sEMG signals. However, a low-frequency peak, contained between the $0-20 \mathrm{~Hz}$ bandwidth, where artefact signal are known to contribute, was consistently observed in power spectra from signals across all horses, muscles and gaits. Therefore, the hypothesis that low-frequency noise contamination would vary depending on muscle and gait was rejected in this study. Qualitative and quantitative analysis of processed signals revealed that application of a $30-40 \mathrm{~Hz}$ cut-off frequency resulted in full attenuation of the low-frequency peak, while maximally retaining the non-artefact portion of the sEMG signal spectra. Therefore, findings from this study support the hypothesis that the attenuation of low-frequency noise in equine sEMG signals requires a higher cut-off frequency than those reported in human literature (De Luca et al., 2010). 
Data from human subjects have revealed that the energetic component of movement artefacts in human sEMG signals generally occurs within the $0-20 \mathrm{~Hz}$ frequency range (van Boxtel, 2001; Clancy et al., 2002; De Luca et al., 2010; Wang et al., 2013), where muscle activation information is minimal and "unstable”, due to unreliable fluctuations of motor unit firing rates (De Luca 2002; De Luca et al., 2010). In De Luca et al. (2010), they adopted a method of deconstructing the human sEMG signal into baseline noise, motion artefact and true sEMG signal components, where movement artefact signals were mainly contained between $0-20 \mathrm{~Hz}$. The dynamic nature of the tasks employed in this equine study meant that it was not possible to induce and analyse isolated movement artefact, as in the De Luca et al. (2010) study. However, the low-frequency peak, which was consistently observed in equine sEMG signals across all horses, muscles and gaits between $0-20 \mathrm{~Hz}$, is in accordance with the spectral component of movement artefact signals from human literature. To the authors' knowledge, this is the first study to illustrate contamination of the low-frequency component of the equine sEMG spectrum, from which HPF cut-off can be analyzed.

An optimal HPF cut-off frequency has been described as a compromise between maximally attenuating noise and artefact contamination, while minimally supressing the low-frequency muscle activation component of the signal (van Boxtel et al., 1998; van Boxtel, 2001; Winter, 2009; De Luca et al., 2010). Visual inspection of power spectral plots in this report consistently showed that a cut-off frequency between $30-40 \mathrm{~Hz}$ attenuated the low-frequency peak, while preserving much of the signal power between 20 - $200 \mathrm{~Hz}$. Uncontaminated human sEMG signals have been reported to exhibit a relatively linear decrease in amplitude with increasing cut-off frequency (De Luca et al., 2010), which was observed in this study from quantitative analysis of RMS signals HPF with $40-80 \mathrm{~Hz}$ cut-offs (Figures $4-7 \mathrm{c}$, e). This suggests that a cut-off $>40 \mathrm{~Hz}$ may result in gradual removal of the low-frequency components of the true equine sEMG signal. Visual examination of sEMG signals, presented in Figures 4 - 7, reveal considerable movement artefact attenuation when HPF filtered with a $40 \mathrm{~Hz}$ cut-off, which was reinforced by significant increases in RMS signal loss. Although continual reduction in movement artefact is evident when 60 and $80 \mathrm{~Hz}$ cut-offs are applied, this reduction was accompanied by significant increases in signal loss across muscles and gaits. This trade-off is especially apparent in 
Figure 7a and provides further support for the use of a $30-40 \mathrm{~Hz}$ cut-off frequency to preserve the true sEMG signal.

In De Luca et al., (2010), increasing HPF cut-off frequencies were found to have a greater effect on the noise component signal (baseline noise plus movement artefact), which experienced signal loss ranging from $69-83 \%$ following application of a $30 \mathrm{~Hz}$ cut-off frequency (De Luca et al., 2010). In comparison, a $30 \mathrm{~Hz}$ cut-off frequency resulted in signal loss ranging from $4-7 \%$ for the true sEMG signal component (De Luca et al., 2010). In this equine study, it was not possible to separate the noise component from sEMG signals; therefore, combined signal loss from the noise and true sEMG components of signals are reported. The degree of signal loss reported by De Luca et al. (2010) for the noise component signal was similarly observed in this study when cut-off frequencies $\geq 40 \mathrm{~Hz}$ were applied to equine sEMG signals (Figure 8). This suggests that application of a $30-40 \mathrm{~Hz}$ cut-off effectively attenuates movement artefact in equine sEMG signals, but may also suppress some of the true sEMG signal. This may be especially relevant for swing phase of TB during trot, which exhibited a comparatively greater rate of signal loss with increasing cut-off intervals (Figure 8c). In contrast, increasing cut-off frequencies resulted in comparatively smaller, albeit significant, increases in signal loss for BF stance phase data during canter (Figure 8b). This finding may indicate that BF signals contain comparatively less energy distribution within the low-frequency range for this task, although further research is required to confirm this.

As hypothesised, the recommended HPF cut-off frequency of $30-40 \mathrm{~Hz}$ for equine sEMG is higher than recommendations from human literature, where systematic investigations have suggested a HPF cut-off frequency of $\geq 20 \mathrm{~Hz}$ (van Boxtel et al, 1998; van Boxtel, 2001; De Luca et al., 2010). However, De Luca et al. (2010) and Wittek et al. (2001) recommend that it may be necessary to increase the 20 Hz cut-off frequency for dynamic applications that exceed the body segment accelerations and loading patterns of general movements. During canter, vertical forces of up to 1.5 times the horses' mass (approximately $500 \mathrm{~kg}$ for an average horse) have been observed (Merkens et al., 1993a). It is therefore 
not surprising that a higher cut-off frequency of $30-40 \mathrm{~Hz}$ was required to remove movement artefact from sEMG signals, obtained during highly dynamic equine gaits.

A cut-off frequency range of $30-40 \mathrm{~Hz}$ is in agreement with previous equine sEMG studies that report using a HPF with a 40 Hz cut-off (Cheung et al., 1998; Kienapfel, 2015; Kienapfel et al., 2018), but do not provide a justification for why this cut-off frequency was chosen. Findings from this study indicate that, while previously employed cut-off frequencies of 10 and $20 \mathrm{~Hz}$ (Hodson-Tole., 2006; Crook et al., 2010; Harrison et al., 2012) are more likely to preserve the low-frequency component of the equine sEMG signal, they are not appropriate for completely attenuating the noise components. It is evident that many equine sEMG studies rely solely on the internal bandwidth of sEMG systems for noise attenuation. However, findings from this study suggest that this may not be appropriate and that additional high-pass filtering is required to achieve full attenuation of low-frequency noise $\leq 20 \mathrm{~Hz}$, as the ideal "brick wall" response of signal filters is not realised. This is illustrated in Figure 9, which demonstrates why filter design must be considered alongside cut-off frequency when evaluating the degree of artefact attenuation. In the current study, a $4^{\text {th }}$ order, zero-lag Butterworth HPF was applied during post-processing, which provides a sharper roll off (steeper transition slope) than the $2^{\text {nd }}$ order HPF with a cut-off frequency of $20 \pm 5 \mathrm{~Hz}$ applied during signal recording. Thus, a residual portion of the signal, which contained movement artefact, was not attenuated completely using the internal bandwidth of the EMG system. This is also evidenced by significant increases in signal loss observed across all muscles and gaits when 20 - $30 \mathrm{~Hz}$ cut-offs were applied post-processing. Determining the optimal filter design (i.e. order) for attenuating low-frequency noise in equine sEMG signals represents an area for future research.

\section{Limitations and future work}

Although this study is the first to provide empirical data for selecting the HPF for processing equine sEMG signals during gait, it focusses on a limited set of conditions and signal processing considerations. Thus, the recommendations for cut-off frequencies reported in this study should be viewed as a starting point for additional research to develop a comprehensive set of equine sEMG signal 
guidelines. In this study, only two equine muscles and gaits were investigated, which cannot be directly extrapolated to other muscle groups and gaits without further empirical data. Additional research is therefore required to determine whether the suggested cut-off of $30-40 \mathrm{~Hz}$ is appropriate across a range of equine muscles, movements and tasks. FL and HL muscles were specifically chosen for this study due to their known functional differences in gait (Payne et al., 2005a,b) and the fact that they are primarily active during stance phase in trot and canter where motion artefact from impacts with the ground are produced. Future work is required to determine the appropriateness of the recommended cut-off frequency for muscles that are primarily active during swing phase, where the source of motion artefact may be more related to changes in velocity.

Although we did not have the opportunity to design this study a priori using a single cohort of animals, we were able to acquire data from $n=5$ horses for each muscle and gait combination studied, which is in accordance with previous sEMG studies employing a sample of $4-6$ horses (i.e. Robert et al., 1999, 2000, 2001a,b, 2002; Hodson-Tole, 2006; Zsoldos et al., 2010a,b). Differences in the size, stride length and velocity between horses also meant that each horse contributed a varying number of stance and swing phases to the data set. This limitation can be addressed in future studies by standardizing the number of strides and velocity for each horse.

Many of the limitations in this study are related to the use of sEMG recording procedures, which may not be optimal. For example, the sensor locations used in this study follow protocols from previous equine studies to promote consistency, rather than because they are optimal. Within the context of the larger development framework for equine sEMG standards, studies to assess the effect of sensor location on noise must also be established. These studies must consider other factors that could not be fully mitigated in this study, such as the impact of cross-talk and innervation zones, which have not been investigated in equine subjects. The choice of a $4^{\text {th }}$ order Butterworth HPF was also based on maintaining consistency with recent equine sEMG studies that specified this filter design (Kienapfel, 2015; Kienapfel et al., 2018) and to elimanate filter design as a confounding factor when studying the 
effects of HPF cut-off. Different types of filters may however be more appropriate for other muscle groups and applications.

Based on findings from this study and the known variation in HPF cut-off across equine sEMG studies (Valentin and Zsoldos, 2016), sufficiently high variability in noise contamination within equine sEMG signals are likely to influence the validity and comparability of findings between studies. Further research is therefore required to understand the effect of HPF on the validity of gait outcome measures. The effect of HPF may be especially important for outcome measures such as muscle activity onset/ offset event detection, where appropriate attenuation of baseline noise and movement artefact are required to increase signal-to-noise ratio and reduce detection of type I errors (Bonato et al., 1998; Merlo et al., 2003; Benedetti et al., 2012).

\section{Conclusion}

Within the context of the larger development framework for equine sEMG standards, this study represents the first in depth examination of HPF filter settings for maximizing the attenuation of movement artefact contamination in equine sEMG data. Findings suggest that a HPF cut-off frequency between $30-40 \mathrm{~Hz}$ is an optimal setting for equine sEMG signals collected from the TB and BF during trot and canter. It is recommended that future equine studies apply appropriate HPF techniques to ensure that outcome measures and their interpretation are obtained from uncontaminated sEMG signals.

\section{Acknowledgements:}

The authors would like to thank the horse owners, riders and handlers for their involvement in this study.

\section{References}

Benedetti, M.G., Agostini, V., Knaflitz, M., Bonato, P., 2012. Muscle activation patterns during level walking and stair ambulation. In: Steele, C. (ed.) Applications of EMG in Clinical Sports Medicine. Rijeka: InTech, 117-126. 
Bonato, P., D’Alessio, T. and Knaflitz, M., 1998. A statistical method for the measurement of muscle activation intervals from surface myoelectric signal during gait. IEEE Transactions in Biomedical Engineering. 45 (3), 287-299. attempt to better represent the signals detected at the intramuscular level. Muscle \& Nerve. 41 (2), pp.234-239.

Cheung, T.K., Warren, L.K., Lawrence, L.M. and Thompson, K.N., 1998. Electromyographic activity of the long digital extensor muscle in the exercising Thoroughbred horse. Equine Veterinary Journal. 30 (3), pp.251-255.

Clancy, E.A., Morin, E.L. and Merletti, R., 2002. Sampling, noise-reduction and amplitude estimation issues in surface electromyography. Journal of Electromyography and Kinesiology. 12 (1), 1-16.

420

Cram, J.R. and Rommen, D., 1989. Effects of skin preparation on data collected using an EMG musclescanning procedure. Biofeedback and Self-regulation. 14 (1), 75-82.

Crook, T.C., Wilson, A. and Hodson-Tole, E., 2010. The effect of treadmill speed and gradient on equine hindlimb muscle activity. Equine Veterinary Journal. 42 (S38), 412-416.

De Luca, C., 1997. The use of surface electromyography in biomechanics. Journal of Applied Biomechanics. 13, 135-163.

De Luca, C.J., 2002. Surface electromyography: Detection and recording. Delsys Incorporated.

De Luca, G., 2003. Fundamental concepts in EMG signal acquisition. Delsys Incorporated.

De Luca, C.J., Gilmore, L.D., Kuznetsov, M., Roy, S.H., 2010. Filtering the surface EMG signal: Movement artifact and baseline noise contamination. Journal of Biomechanics. 43 (8), 1573-1579.

Harrison, S.M., Whitton, R.C., King, M., Haussler, K.K., Kawcak, C.E., Stover, S.M. and Pandy, M.G., 2012. Forelimb muscle activity during equine locomotion. Journal of Experimental Biology. 215 (17), 2980-2991. 
Hermens, H.J., Freriks, B., Disselhorst-Klug, C., Rau, G., 2000. Development of recommendations for SEMG sensors and sensor placement procedures. Journal of Electromyography and Kinesiology. 10 (5), 361-374.

Hjerten, G., Drevemo, S. and Eriksson, L.E., 1994. Shortening of the hind limb in the horse during the stance phase. Equine Veterinary Journal. 26 (S17), 48-50.

Hodson-Tole, E., 2006. Effects of treadmill inclination and speed on forelimb muscle activity and kinematics in the horse. Equine and Comparative Exercise Physiology. 3 (2), 61-72.

Holt, D., St George, L.B., Clayton, H.M. and Hobbs, S.J., 2017. A simple method for equine kinematic gait event detection. Equine Veterinary Journal. 49 (5), 688-691

Huigen, E., Peper, A. and Grimbergen, C.A., 2002. Investigation into the origin of the noise of surface electrodes. Medical and Biological Engineering and Computing. 40 (3), 332-338.

Kienapfel, K., 2015. The effect of three different head-neck positions on the average EMG activity of three important neck muscles in the horse. Journal of Animal Physiology and Animal Nutrition. 99 (1), $132-138$

Kienapfel, K., Preuschoft, H., Wulf, A., Wagner, H. 2018. The biomechanical construction of the horse's body and activity patterns of three important muscles of the trunk in the walk, trot and canter. Journal of Animal Physiology and Animal Nutrition. 102 (2), pp.e818-e827.

Merkens, H.W., Schamhardt, H.C., Osch, G.J. and Bogert, A.V.D., 1993a. Ground reaction force patterns of Dutch Warmblood horses at normal trot. Equine Veterinary Journal. 25 (2), pp.134-137.

Merkens, H.W., Schamhardt, H.C., Van Osch, G.J. and Hartman, W., 1993b. Ground reaction force patterns of Dutch Warmbloods at the canter. American Journal of Veterinary Research. 54 (5), 670674.

Merletti, R., 1999. Standards for reporting EMG data. Journal of Electromyography and Kinesiology. 9 (1), 3-4.

475 Merlo, A., Farina, D. and Merletti, R., 2003. A fast and reliable technique for muscle activity detection from surface EMG signals. IEEE Transactions on Biomedical Engineering. 50 (3), 316-323. 
Payne, R.C., Hutchinson, J.R., Robilliard, J.J., Smith, N.C. and Wilson, A.M., 2005a. Functional specialisation of pelvic limb anatomy in horses (Equus caballus). Journal of Anatomy. 206 (6), 557574.

Payne, R.C., Veenman, P. and Wilson, A.M., 2005b. The role of the extrinsic thoracic limb muscles in equine locomotion. Journal of Anatomy. 206 (2), 193-204.

Peham, C. and Schobesberger, H., 2006. A novel method to estimate the stiffness of the equine back. Journal of Biomechanics. 39 (15), 2845-2849.

Potvin, J.R. and Brown, S.H.M., 2004. Less is more: high pass filtering, to remove up to 99\% of the surface EMG signal power, improves EMG-based biceps brachii muscle force estimates. Journal of Electromyography and Kinesiology. 14 (3), 389-399.

Robert, C., Audigié, F., Valette, J.P., Pourcelot, P. and Denoix, J.M. 2001a. Effects of treadmill speed on the mechanics of the back in the trotting saddlehorse. Equine Veterinary Journal. 33 (S33), pp.154159.

495

Robert, C., Valette, J.P., Degueurce, C. and Denoix, J.M. 1999. Correlation between surface electromyography and kinematics of the hindlimb of horses at trot on a treadmill. Cells Tissues Organs. 165 (2), pp.113-122.

Robert, C., Valette, J.P. and Denoix, J.M. 2000. The effects of treadmill inclination and speed on the activity of two hindlimb muscles in the trotting horse. Equine Veterinary Journal. 32 (4), pp.312-317.

Robert, C., Valette, J.P. and Denoix, J.M. 2001b. The effects of treadmill inclination and speed on the activity of three trunk muscles in the trotting horse. Equine Veterinary Journal. 33 (5), pp.466-472.

Robert, C., Valette, J.P., Pourcelot, P., Audigie, F. and Denoix, J.M. 2002. Effects of trotting speed on muscle activity and kinematics in saddlehorses. Equine Veterinary Journal. 34 (S34), pp.295-301.

Schuurman, S.O., Kersten, W. and Weijs, W.A., 2003. The equine hind limb is actively stabilized during standing. Journal of Anatomy. 202 (4), 355-362.

Stegeman D.F. and Hermens H.J. 1998. Standards for surface electromyography: The European project (SENIAM). In: Hermens H.J., Rau G., Disselhorst-Klug C., Freriks B. (eds.) Surface Electromyography 
515 Application Areas and Parameters. Proceedings of the Third General SENIAM Workshop on Surface Electromyography. Aachen, Germany. pp. 108-112.

Valentin, S. and Zsoldos, R.R., 2016. Surface electromyography in animal biomechanics: A systematic review. Journal of Electromyography and Kinesiology, 28, 167-183.

520

van Boxtel, A., 2001. Optimal signal bandwidth for the recording of surface EMG activity of facial, jaw, oral, and neck muscles. Psychophysiology, 38 (1), 22-34.

van Boxtel, A., Boelhouwer, A.J.W. and Bos, A.R., 1998. Optimal EMG signal bandwidth and 525 interelectrode distance for the recording of acoustic, electrocutaneous, and photic blink reflexes. Psychophysiology. 35 (6), 690-697.

van Weeren, P. R., van den Bogert, A. and Barneveld, A. 1990a. Quantification of skin displacement in the proximal parts of the limbs of the walking horse. Equine Veterinary Journal. 22 (S9), 110-118.

530

van Weeren, P.R., van den Bogert, A.J. and Barneveld, A., 1990b. A quantitative analysis of skin displacement in the trotting horse. Equine Veterinary Journal. 22 (S9), 101-109.

Wang, J., Tang, L. and Bronlund, J.E., 2013. Surface EMG signal amplification and filtering. International Journal of Computer Applications. 82 (1), 15-22.

Williams, J.M., Johnson, C., Bales, R., Lloyd, G., Barron, L. and Quest, D., 2014. Analysis of Temporalis and Masseter adaptation after routine dental treatment in the horse via surface electromyography. Comparative Exercise Physiology. 10 (4), 223-232.

540

Winter, D., 2009. Biomechanics and Motor Control of Human Movement. 4th ed. Hoboken, NJ: John Wiley and Sons Inc.

Winter, D., Rau, G., Kadefors, R., Broman, H., De Luca, C., 1980. Units, terms and standards in 545 reporting of EMG research. In: Report by the Ad Hoc Committee of the International Society of Electrophysiological Kinesiology.

Wittek, A., Ono, K., Kajzer, J., Ortengren, R. and Inami, S., 2001. Analysis and comparison of reflex times and electromyogram of cervical muscles under impact loading using surface and fine-wire electrodes. IEEE Transactions on Biomedical Engineering. 48 (2), 143-153. 
Zaneb, H., Kaufmann, V., Stanek, C., Peham, C. and Licka, T.F., 2009. Quantitative differences in activities of back and pelvic limb muscles during walking and trotting between chronically lame and nonlame horses. American Journal of Veterinary Research. 70 (9), 1129-1134.

555

Zsoldos, R.R., Kotschwar, A.B., Kotschwar, A., Groesel, M., Licka, T. and Peham, C. 2010a. Electromyography activity of the equine splenius muscle and neck kinematics during walk and trot on the treadmill. Equine Veterinary Journal. 42 (S38), 455-461.

560 Zsoldos, R.R., Kotschwar, A., Kotschwar, A.B., Rodriguez, C.P., Peham, C. and Licka, T. 2010b. Activity of the equine rectus abdominis and oblique external abdominal muscles measured by surface EMG during walk and trot on the treadmill. Equine Veterinary Journal. 42 (S38), pp.523-529.

Zsoldos, R.R., Krüger, B. and Licka, T.F., 2014. From maturity to old age: tasks of daily life require a 565 different muscle use in horses. Comparative Exercise Physiology. 10 (2), 75-88.

570 


\section{Captions to Figures}

Figure 1a) anatomical kinematic marker locations used to create sagittal plane angle for hindlimb hoof impact and lift-off gait events: 1 , hind distal interphalangeal joint; 2 , metatarsalphalangeal joint; 3 , talus. Kinematic marker attached over croup for stride velocity calculation (4). b) anatomical kinematic marker locations used to create sagittal plane angle for forelimb hoof impact and lift-off gait events: 4, fore distal interphalangeal joint; 5, metacarpalphalangeal joint; 6 , lateral epicondyle of humerus. sEMG sensors applied over 8, Triceps Brachii and 9, Biceps Femoris muscles.

Figure 2. sEMG signal from Biceps Femoris during canter illustrating HL hoof impact (green line) and hoof lift-off (red line) events. Stance and swing phase are illustrated by blue and purple shaded areas, respectively. Note: signal is DC offset removed and high-pass filtered (Butterworth 4th order) with a $50 \mathrm{~Hz}$ cut-off, which was chosen at random to illustrate the phasic activity pattern of the signal in relation to the kinematic gait events.

605

Figure 3. RMS signal loss and residual signal (\%) data from sEMG signals obtained from the Triceps Brachii during one stance and swing phase at trot.

Figure 4. a.) Representative sEMG signals from Biceps Femoris from one horse during canter. Data are time normalised across one canter stride. Green and red vertical lines represent kinematic HL hoof impact and lift-off events, respectively. Corresponding power spectra (b. and d.) and RMS enveloped signals (c and e) for stance and swing phase data.

Figure 5. a.) Representative sEMG signals from Triceps Brachii from one horse during canter. Data are time normalised across one canter stride. Green and red vertical lines represent kinematic FL hoof impact and lift-off events, respectively. Corresponding power spectra (b. and d.) and RMS enveloped signals (c and e) for stance and swing phase data.

Figure 6. a.) Representative sEMG signals from Biceps Femoris from one horse during trot. Data are time normalised across one trot stride. Green and red vertical lines represent kinematic HL hoof impact and lift-off events, respectively. Corresponding power spectra (b. and d.) and RMS enveloped signals (c and e) for stance and swing phase data. 
Figure 7. a.) Representative sEMG signals from Triceps Brachii from one horse during trot. Data are 625 time normalised across one trot stride. Green and red vertical lines represent kinematic FL hoof impact and lift-off events, respectively. Corresponding power spectra (b. and d.) and RMS enveloped signals (c and e) for stance and swing phase data.

Figure 8. Mean \pm sd RMS signal loss (\%) (solid line) and residual signal (\%) (dashed line) for stance 630 (blue lines) and swing phase (orange lines) from Biceps Femoris during a.) trot and b.) canter and Triceps Brachii during c.) trot and d.) canter.

Figure 9. Magnitude response of Butterworth high-pass filters with a $20 \mathrm{~Hz}$ cut-off frequency illustrating the relative cut-off sharpness of varying orders $(n=1,2,4,8)$. Adapted from De Luca 635 (2003). 

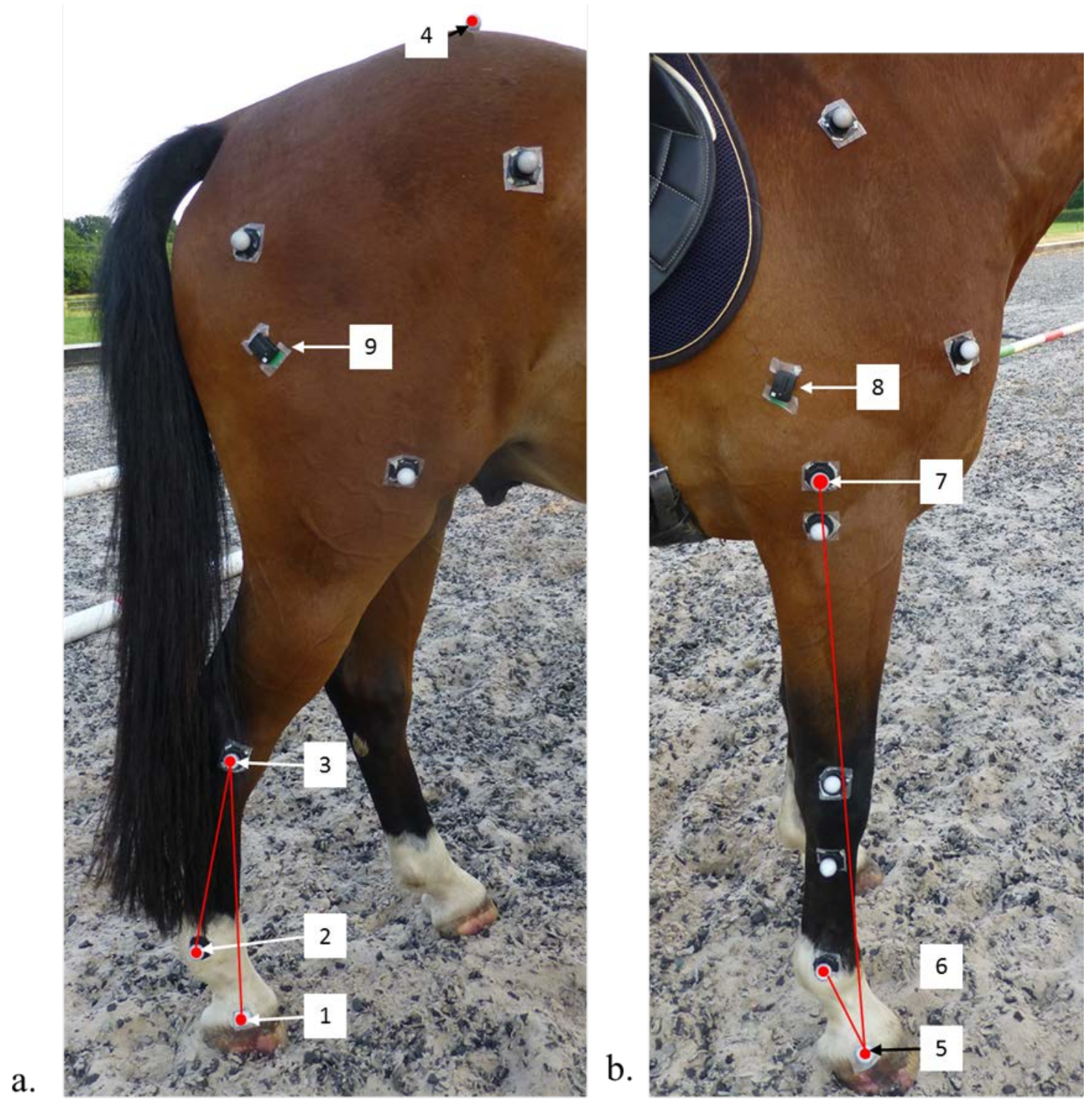

Figure 1. 


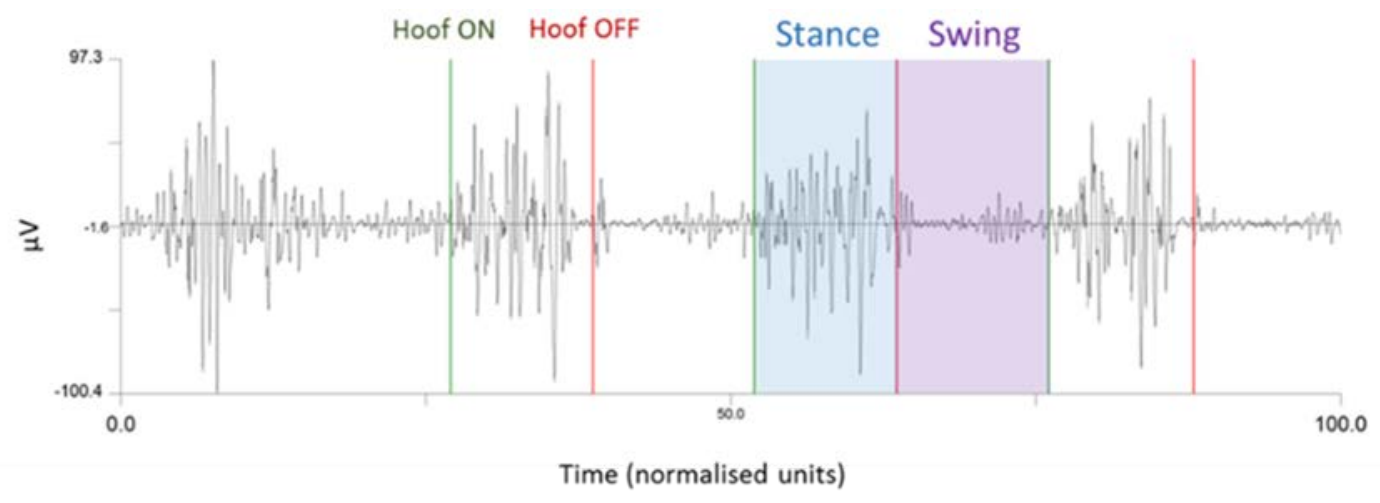

650

Figure 2

655 


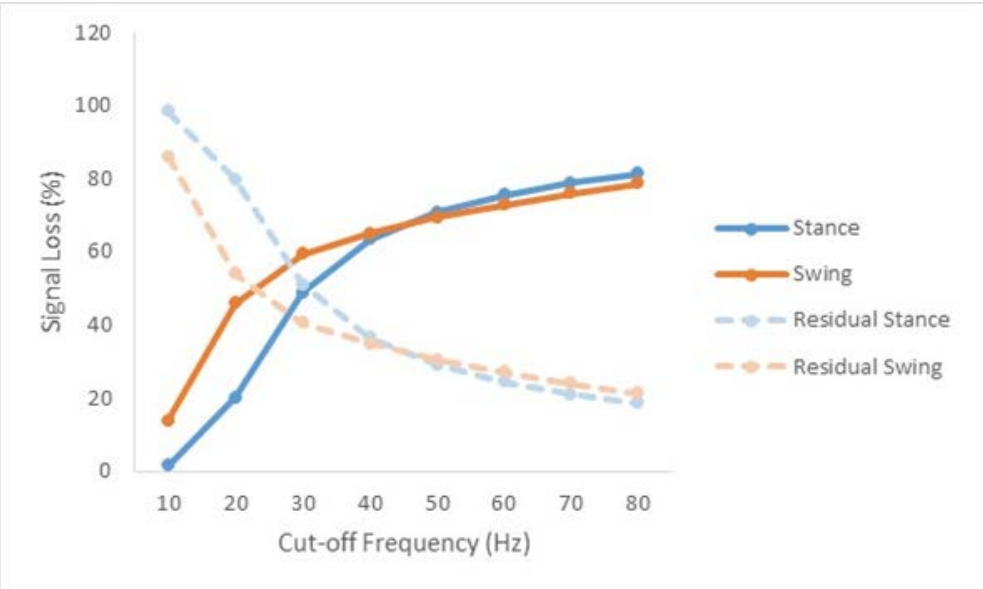

Figure 3.

680

685

690

695 


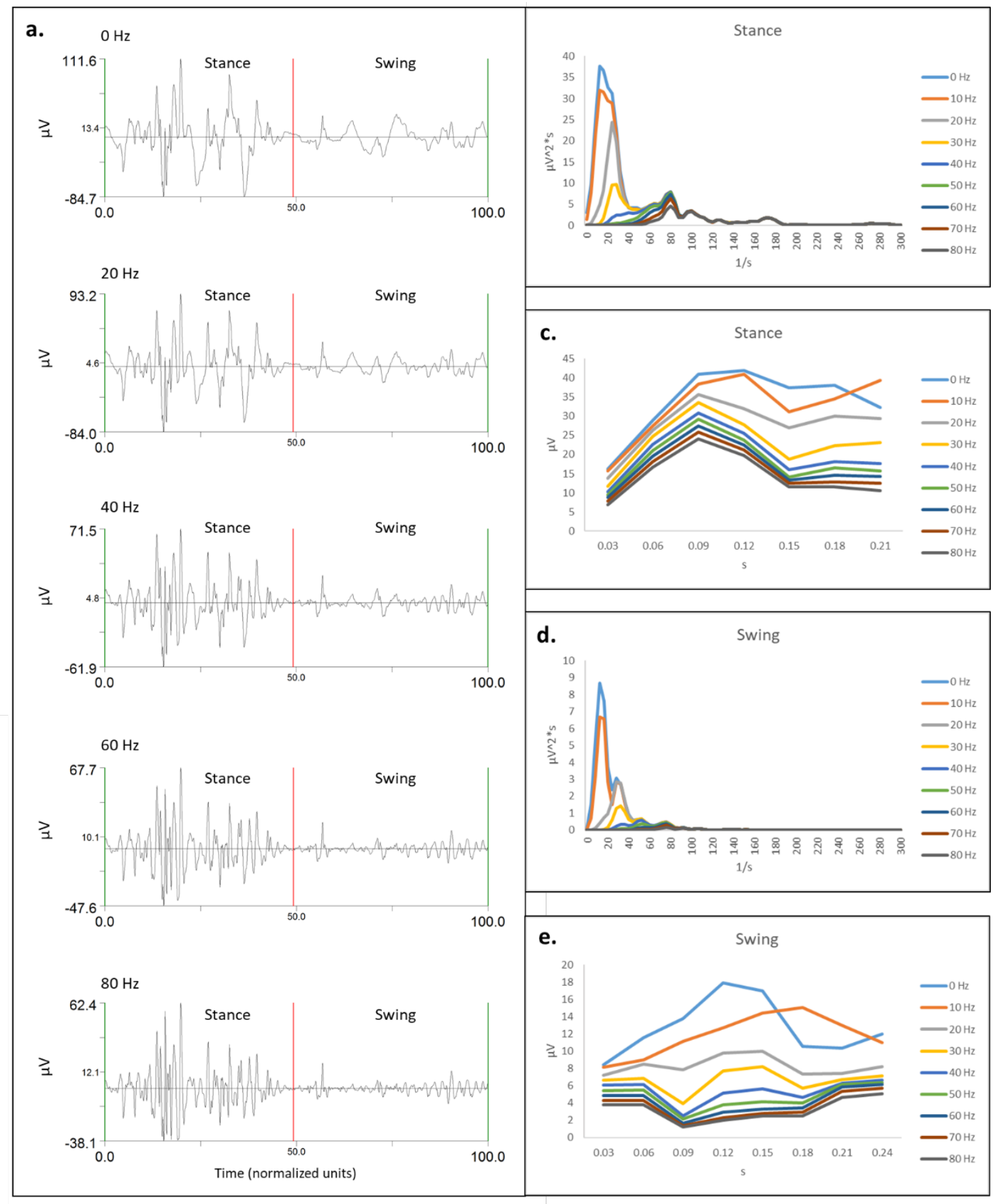

Figure 4 


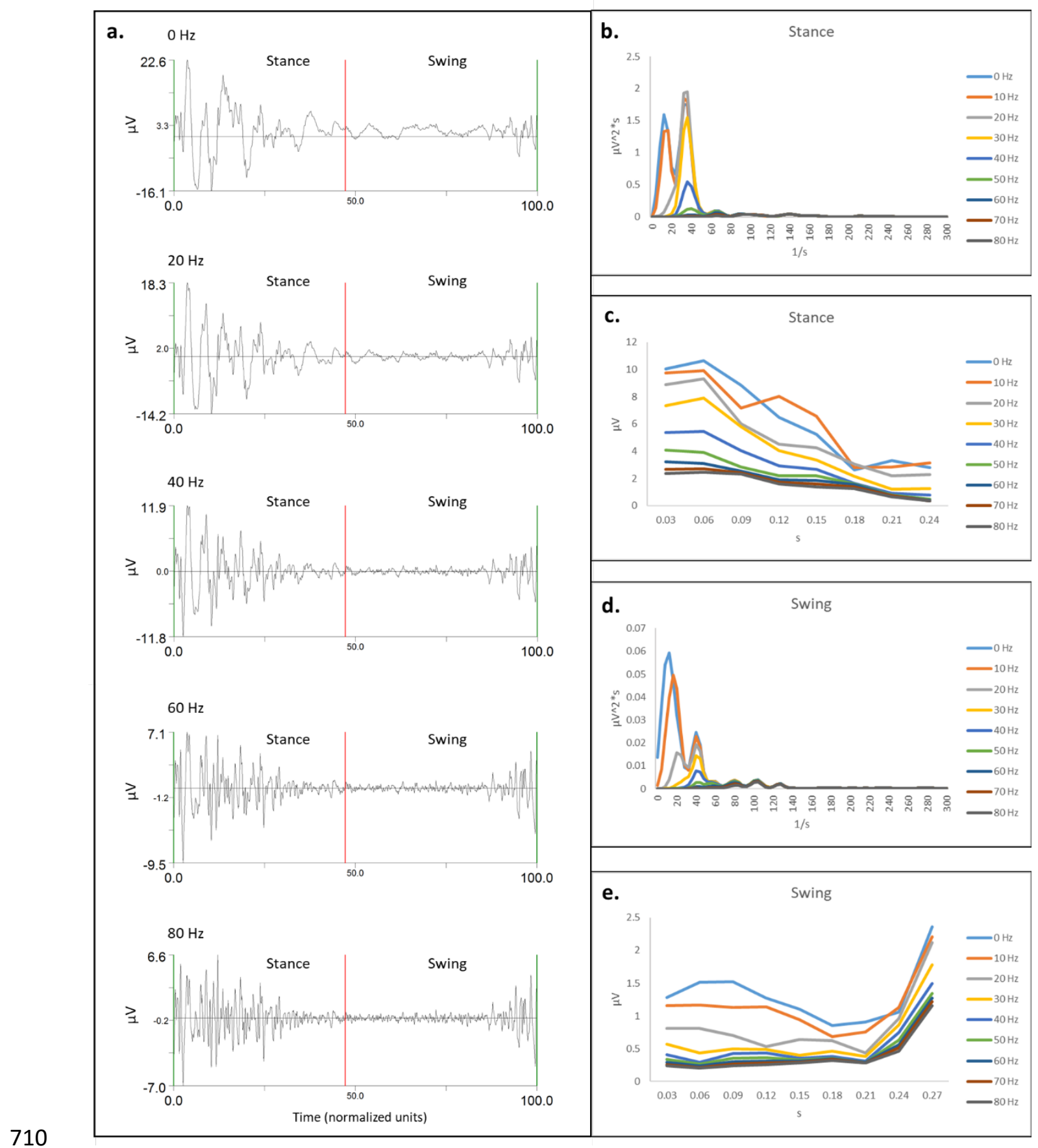

Figure 5 


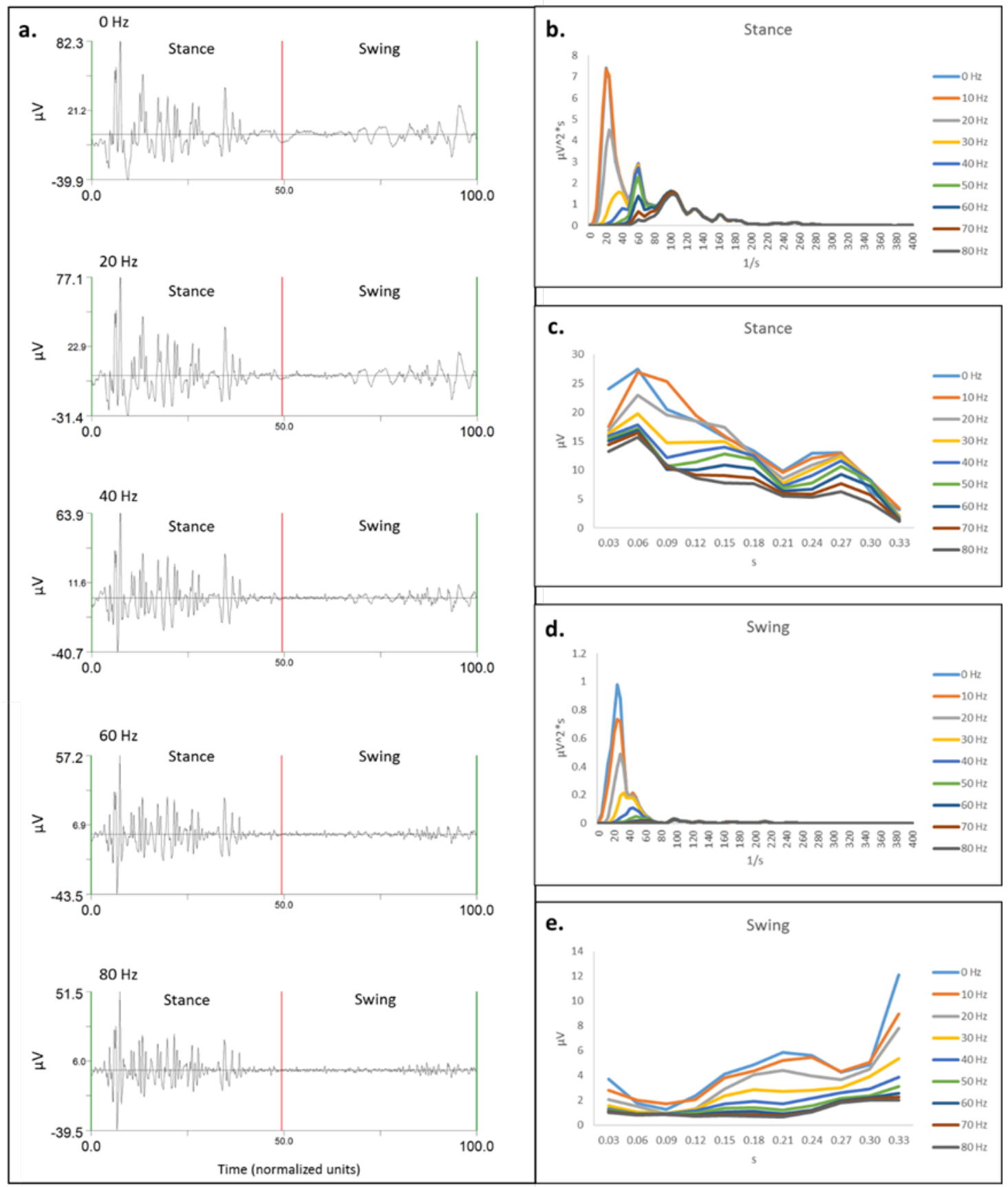

Figure 6 


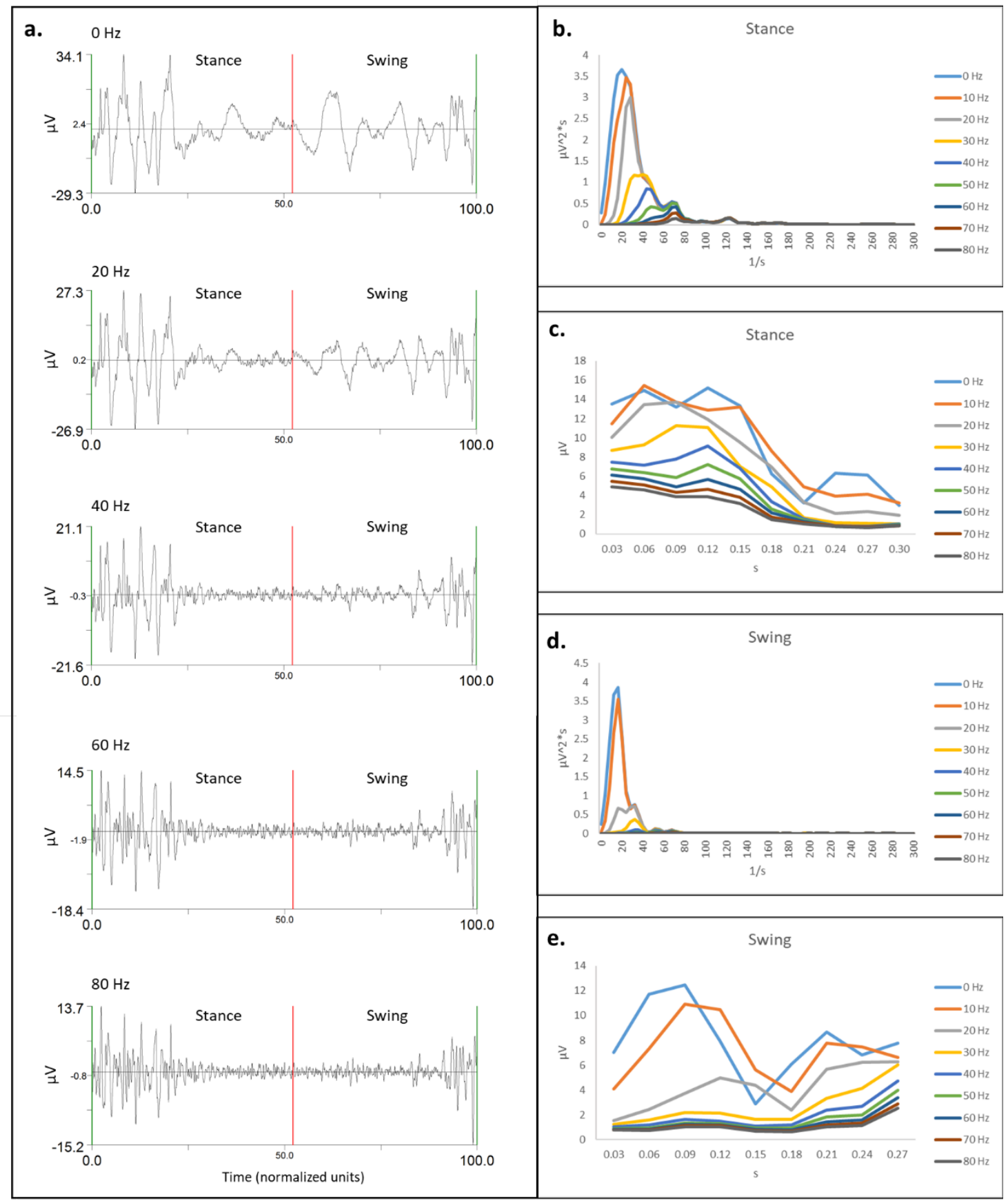

Figure 7 

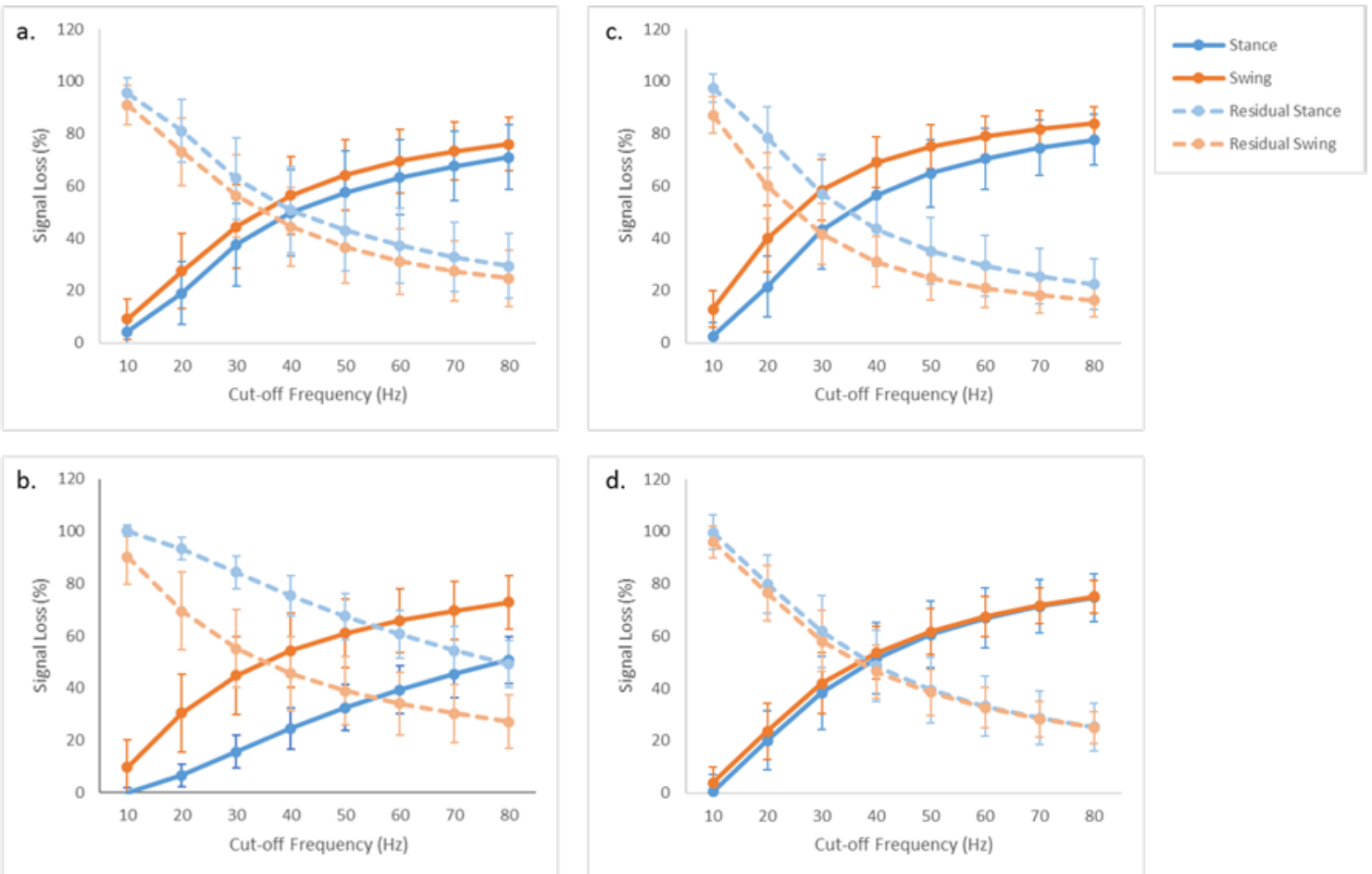

Figure 8 


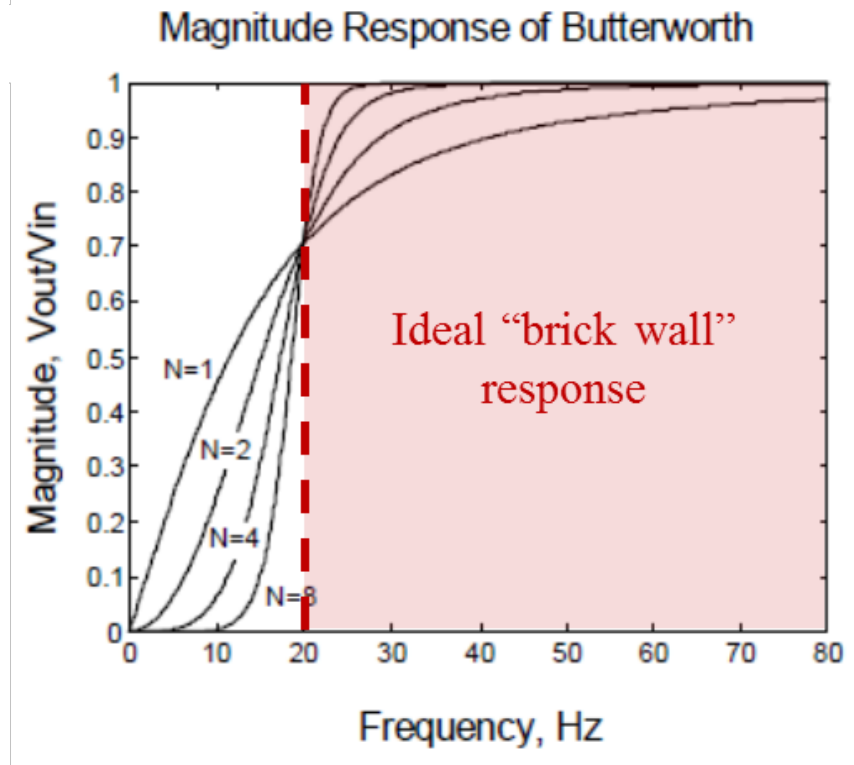

Figure 9 
Table 1. Mean difference (MD), 95\% confidence intervals (95\% CI) and p values (P) for mean RMS signal loss (\%) data between increasing $10 \mathrm{~Hz}$ cut-off frequency increments. *Significant difference between mean RMS signal loss (\%) data for increasing $10 \mathrm{~Hz}$ cut-off frequency increments (i.e. $10 \mathrm{~Hz}$ RMS signal loss vs. $20 \mathrm{~Hz}$ RMS signal loss) for the corresponding muscle, gait and stance or swing phase combination.

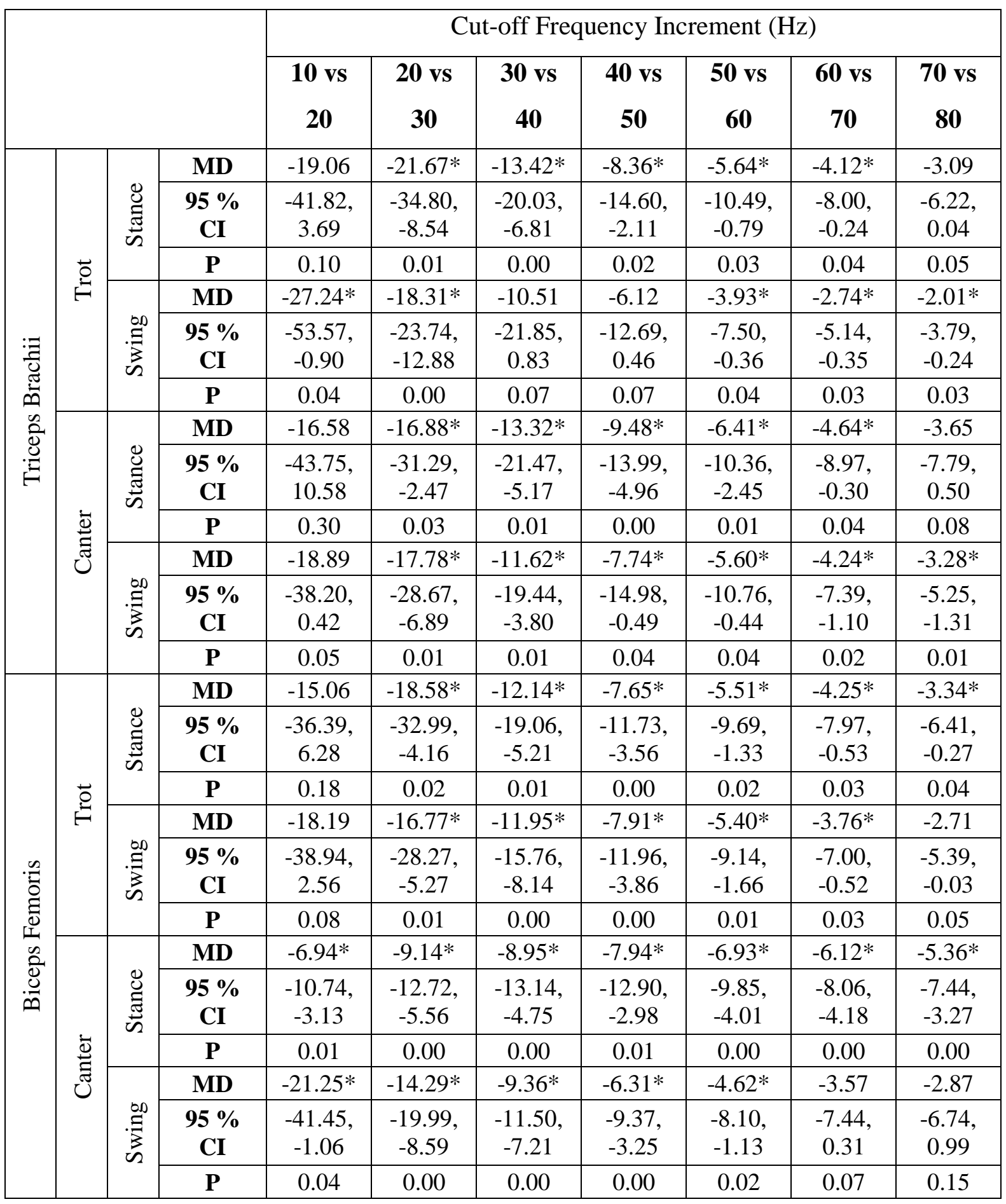

